\title{
Apuntes para una \\ Historia del Psicoanálisis en Argentina
}

\author{
HORACIO R. MARÍN
}

\section{RESUMEN}

Se trata de trazar un bosquejo de la historia del pensamiento psiquiátrico, así como de las instituciones que se fueron creando, analizando sus relaciones ideológicas y político-sociales, esenciales para el desarrollo de unas $u$ otras corrientes de una disciplina médica que tiene unas importantes implicaciones sociales.

\begin{abstract}
It's intended to trace an outline of the history of the psychiatric Argentinean thought through their more important thinkers, as well as through the institutions that were created during the period, analyzing their ideological and socio-political relationships, essential for the development of a medical discipline that has very important social implications.
\end{abstract}

\section{Introducción}

Para escribir acerca del Psicoanálisis y su impacto en América, es imprescindible seguir la trayectoria de Freud y su relación con el llamado nuevo continente. En América del Sur, José Ingenieros malogró tempranamente la importancia del nuevo método terapéutico superador de la hipnosis y la catarsis, así como también impidió traer a la Argentina la nueva manera de comprender la psique y desarrollar la investigación por medio de la revolución epistemológica que se estaba gestando en Viena. En los EE.UU. James Put- 
nam había creado la primera sociedad psicoanalítica. Putnam, viejo médico y amante de la filosofía, logró que el maestro vienés «les llevase la peste». Mientras tanto, en Buenos Aires, Ingenieros, que practicaba la psiquiatría, escribía en demasía sin llegar a ser un filósofo y, además, era socialista. En los primeros tramos de este siglo, el socialismo argentino, como el de otras partes del mundo, era "progresista», estando ligado a los criterios de evolución positivista imperantes en la época.

Ingenieros había tenido la oportunidad de acceder a las «leçons du mardi" de Charcot y miró con desprecio los novedosos aportes de Freud, adhiriéndose a la terapia de la hipnosis y la sugestión, que puso en práctica junto a José María Ramos Mejía, en el Hospital de San Roque, de Buenos Aires. Ingenieros prefirió la sugestión a la hipnosis, desde el punto de vista de la psicoterapia. Así como prefería a Berheim antes que a Liebault. Por otra parte, Ingenieros logró que Babinski se interesara por algunas observaciones clínicas suyas, en abril de 1906.

En la revista La Semana Médica, en 1904, en una nota firmada por Luis Agote, podía observarse cierto triunfalismo que parecía reflejar el denominado «ser nacional». En efecto, Agote escribió:

\footnotetext{
«En cuanto al valor que le diera Babinski - a su signo-, valor que ha sido aceptado sin discusión por la mayoría de los autores que han estudiado el punto, ha sufrido últimamente un rudo golpe con la presentación de un caso de hemiplejía histérica hecha por el doctor Ingenieros» ${ }^{1}$.
}

El signo que Babinski había comunicado en sólo treinta líneas a la Sociedad de Biología de París el 22 de febrero de 1896, había sido rebatido por el polifacético Ingenieros.

Más allá de las modestias iniciales de Babinski y las fanfarrias rioplatenses, producidas en un país que se acercaba - lleno de contradicciones- orgulloso, a su primer centenario de la independencia, dijo Ingenieros:

«El Profesor Babinski nos hizo el honor de dedicar una de sus lecciones clínicas (abril 1906) en el Hospital de la Pitié, de París, al análisis del presente capítulo (Ausencia de los síntomas de la histérica y presencia de los propios de la orgánica; valor del signo de Babinski) aceptando que estos hechos excepcionales, aunque atenúan el valor absoluto de su signo, no le quitan su importancia como regla general ${ }^{2}$.

\footnotetext{
1 La Semana Médica (1904), 10 de marzo, Buenos Aires.

2 Ingenieros, José (1960): Histeria y Sugestión. Ed. Roggero-Ronal. Buenos Aires, p. 84. La primera edición data de 1904.
} 
Dicho en buen romance, Babinski complacía al joven visitante, pero seguía firme en sus observaciones.

El joven Ingenieros abarcará - para mi gusto- demasiados temas. En 1906 contaba con 29 años. Se había graduado como doctor en Medicina en 1900. Su trabajo de tesis fue laureado y editado simultáneamente en Italia y Argentina, en 1903.

En estos años, por la influencia negativa de figuras del campo de la cultura, la medicina y la política -e Ingenieros es un buen ejemplo de ello-, sumada también la acción de la sociedad médica porteña, el Psicoanálisis no penetró en la intelectualidad rioplatense de la primera década del siglo.

Uno de los más lúcidos y originales pensadores argentinos (también médico, psiquiatra y socialista) Alejandro Korn (1860-1936), escritor y poeta, no se interesó - por lo que se conoce - en la obra de Freud. Conozcamos algo de su pensamiento en este fragmento de su obra La Libertad Creadora:

«El sujeto: sin embargo, distingo entre los actos propios y los impuestos. El psicólogo: esa es una opinión subjetiva. Es un detalle insignificante.

El sujeto: además tengo propósitos y proyectos que quiero realizar.

El psicólogo: deseos y quimeras que usted se forja al margen de los hechos (el psicólogo experimental le habla de la "exactitud objetiva de la experimentación científica").

El sujeto: admirable ciencia. Pero si renuncio a mi libertad de la que vivo tan convencido, ¿qué me queda?

El psicólogo: nada (...) en realidad usted no es nadie. Ni siquiera es una hipótesis.

El sujeto: respete mis fueros. Yo no soy una muñeca, soy el sujeto libre...n ${ }^{3}$

Este diálogo imaginario, por momentos brillante, para mi gusto, de este anti-positivista, idealista gnoseológico inspirado en Kant, revela un respeto epistemológico por otros discursos que estaban lejos de ser hegemónicos en el momento de ser redactado.

Si bien Korn discute a su veż con algún contradictor imaginario, acerca del monismo o del dualismo de la conciencia, no deja de ser interesante la siguiente reflexión:

«La unidad psíquica, la sensación, nunca es simple, menos aún lo son los estados de ánimo. Ni en el dominio de lo objetivo, ni en el de lo subjetivo, podemos fijar una unidad. Tampoco lo es el yo, ni lo es el objeto intuido» ${ }^{4}$.

\footnotetext{
${ }^{3}$ Korn, A. (1963): La Libertad Creadora. Ed. Claridad. Buenos Aires, pp. 84-87.

${ }^{4}$ Korn, A. (1938): Obras. Ensayos Filosóficos. Ed. Universidad Nacional de La Plata. Vol. I, pp. 46-47. 
Korn no habla de la Ichspaltung freudiana, como tampoco de la represión, como piedra angular del edificio analítico, pero qué cerca rondaba de estos conceptos, en tiempos contemporáneos a Freud, desde otras vertientes del pensamiento.

El presunto desinterés u omisión de Alejandro Korn por la obra de Freud trasladaría la cuestión del psicoanálisis en la Argentina a varias décadas más tarde. Esta carencia en el pensamiento no sería ocupada por ninguna persona, seriamente, hasta la década de los cuarenta. Existen algunas adhesiones que no fueron suficientes para la constitución de una institución psicoanalítica, o para organizar un pensamiento psicoanalítico sin institución ${ }^{5}$.

En 1910, tuvo lugar en Buenos Aires el Congreso Médico Internacional, donde G. Greve, un médico alemán, presentó la ponencia titulada: Sobre psicología y psicoterapia de ciertos estados angustiosos ${ }^{6}$.

El apego argentino hacia cuestiones neuropatológicas, como también hacia el alienismo y la psiquiatría forense abrieron una brecha entre las ideas freudianas y la práctica de neurólogos y psiquiatras de la época. La ponencia de Greve, en la Sección de Neurología, Psiquiatría, Antropología y Medicina Legal del Congreso, pasó inadvertida. No sería así en EE.UU. donde la introducción del psicoanálisis fue realizada de un modo particularmente notable.

Quizá la revolución rusa de 1917 tuvo cierto impacto en el pensamiento psiquiátrico argentino, lo que no facilitó el surgimiento del psicoanálisis. Fue más benevolente con el pensamiento freudiano el mismo Trotsky — cuando declinaba su posición política frente a Stalin-que los psiquiatras argentinos. Ingenieros, como tantos intelectuales de la época, realizaba comentarios admirativos acerca de «los tiempos nuevos». En tanto. Ingenieros extendía una suerte de "cheque en blanco" a la Revolución y a su producción, de la cual devendría Pavlov como arquetipo científico en psicología, el revolucionario escribía:

«el intento de declarar al Psicoanálisis incompatible con el marxismo, volviéndole la espalda sin ceremonia al freudismo es demasiado simplista (...) en ningún caso estamos obligados a adoptar el freudismo. Se trata de una hipótesis de trabajo que puede dar - y que está dando, indiscutiblemente- hipótesis y conclusiones que se inscriben en la línea de la psicología materialista. La vía experimental lleva, en su momento, a la prueba. Pero aunque fuese menos segura, no tenemos ni derecho a prohibir otra vía que se esfuerza por anticipar conclusiones a las que conduce la vía experimental mucho más len-

\footnotetext{
${ }^{5}$ Un ejemplo es la tesis de doctorado de Agrelo, del año 1908, Psicoterapia y reeducación psíquica. El padrino de tesis fue Ingenieros. En el trabajo existen conceptos cercanos al psicoanálisis, si bien no figura el nombre de Freud.

${ }^{6}$ Freud destacó esta participación en su Historia del Movimiento Psicoanalítico.
} 


\section{APUNTES PARA UNA HISTORIA DEL PSICOANALISIS EN ARGENTINA}

tamente (...) si ya resulta que en la edificación económica no pueden resolverse los problemas con simples órdenes, hay que aprender a comerciar, en las ciencias el método de ordeno y mando sólo puede llevar al prejuicio y a la vergüenza. En este campo, hay que aprender a aprender» ${ }^{7}$.

Los escritos de Trotsky fueron realizados en plena guerra interna con Stalin. Por lo que se desprende de la lectura del texto, existe una semejanza al "prohibido prohibir» de mayo del 68 de París, y una invitación a la defensa del pluralismo científico para acrecentamiento cultural del proletariado.

Contemporáneamente a los pensamientos de Trotsky, en Buenos Aires, el neuropsiquiatra español Gonzalo R. Lafora dictaba, en el año 1923, una serie de conferencias sobre el psicoanálisis.

Hasta 1923, Freud había sido presentado en ámbitos científicos desde un lugar de desacreditación absoluta, a excepción del alemán Greve, del cual no se conoce trayectoria después del Congreso de 1910.

También en 1923, Aníbal Ponce, con el seudónimo de «Luis Campos Aguirre», publicaba en la Revista de Filosofía el trabajo: "La divertida estética de Freud», ácido análisis, de espíritu bromista, en el cual subyace de nuevo el positivismo ${ }^{8}$. Escribía Ponce en el artículo:

«Pero debí esperar a que apareciese la Introducción al Psicoanálisis para que pudiera comprender el significado profundamente cómico de la obra magna del gran médico humorista (...) el pansexualismo es la idea fija del freudismo. Así la nutrición -que para la biología entera es el acto primordial-, aparece aquí como un fenómeno secundario del Deseo universal»" ${ }^{9}$.

A pesar suyo Ponce planteaba el fenómeno de la succión como una humorada deseante.

Ponce, al igual que Lafora, criticaba las relaciones entre la estética y el psicoanálisis. Es sabido que al propio Freud no le satisfacían demasiado sus propias conclusiones sobre el fenómeno de la sublimación.

Freud jamás fue tan lineal en lo que denominó "psicoanálisis aplicado» (Vgr. Moisés y el monoteísmo). Jamás renegó del cuerpo, de la biología hecho que Trotsky advirtió-, expuso su teoría acerca de las series complementarias, y se dedicó a construir su teoría como una metapsicología, como

\footnotetext{
${ }^{7}$ Tomado de Cuaderno del PRT (Partido Revolucionario de los Trabajadores) (1970): "Apuntes de Estética y Política».

${ }^{8}$ Aníbal Ponce (1898-1938), sociólogo y ensayista. Fundador de la revista Dialéctica, de orientación marxista. Se exilió en México durante la década infame de gobiernos conservadores.

${ }^{9}$ Revista de Filosofía, Año IX, N. ${ }^{\circ} 1$, p. 101.
} 
otra forma de entender la clínica, como una forma de psicoterapia causal y un método de investigación.

En 1930, el médico argentino Jorge Thénon envió un libro de su autoría a Freud: Psicoterapia comparada y psicogénesis. Aquí se malogra otra oportunidad para el advenimiento del pensamiento freudiano, ya que a pesar de que Freud responderá el 25 de agosto del mismo año en forma positiva, Thénon se apartará del camino que él mismo había buscado para consagrarse a la Psicología Dialéctica, en especial desde $1935^{10}$. Creo que el joven Thénon no se arriesgó a seguir mostrando su producción a Freud, más allá de su viraje ideológico, en momentos en que en Alemania y Austria todavía existían nutridos grupos de intelectuales que pugnaban por acercar el marxismo al psicoanálisis y viceversa.

En los años treinta, buena parte de la información psicoanalítica llegaba a Buenos Aires a través de la obra de divulgadores. Los libros de «vulgarización» constituían una fuente de conocimiento mal transmitido, debido a los innumerables errores de autor y de la casa editorial. De todos modos, las publicaciones de un autor de la época, Gómez Nerea, que transcribía párrafos enteros de la traducción de Freud realizada por López Ballesteros, junto a otros, producto de su pluma e imaginación, fue una presencia infaltable en las bibliotecas familiares de la clase media urbana argentina, hasta entrados los años cincuenta ${ }^{11}$. Cabe una reflexión por la sincronía en la que se producían estos textos aclaratorios de la «vasta obra de Freud»... ¿Cómo habría sido confeccionada la versión que le pareció - irresponsablemente divertida - ridícula a Aníbal Ponce, en 1923?

En América Latina el psicoanálisis no sólo debió confrontarse con los adherentes a la reflexología, sino también con profesionales simpatizantes de la fenomenología y de un psicoanálisis no freudiano, vale decir, junguiano. La introducción de dos autores con los que Freud había roto lazos, Adler y Jung, se debe a la obra de los peruanos Honorio Delgado y Mariano Ibérico, quienes realizaron un excelente trabajo en Lima, Perú, en 1933 y 1936, fecha de dos ediciones sucesivas de su libro Psicologia, de gran difusión en Buenos Aires.

Hasta ese momento, fines de la década de los treinta, vemos cómo el psicoanálisis se había expandido territorialmente, en especial en los países de

${ }^{10}$ La carta de Freud a Thénon se encuentra publicada en la revista La Semana Médica (1933), Buenos Aires.

${ }^{11}$ La obra de Freud fue autorizada para su publicación en castellano en 1923, en Biblioteca Nueva en 17 volúmenes. Esta edición, como también sus sucesivas reediciones fueron fundamentales para algunos colegas franceses. Esta observación me la hizo conocer un instructor de residentes en París, en el Hospital Henri-Rousselle, del complejo hospitalario Saint-Anne, en enero de 1987. Todavía la edición de López Ballesteros es fuente de consulta en ese lugar. 


\section{APUNTES PARA UNA HISTORIA DEL PSICOANALISIS EN ARGENTINA}

habla inglesa, con tendencias a la retracción y/o desaparición en los países que luego conformarían el Eje, con zonas de exclusión en la Unión Soviética y áreas de influencia en América del Sur.

\section{El Psicoanálisis en Argentina}

Para el Departamento de Historia del Psicoanálisis de la Asociación Psicoanalítica Argentina, coordinado en 1982 por los Dres. Jorge Mom, Gilda Foks y Juan Carlos Suárez, la década de los treinta se caracteriza por la extensión y difusión del psicoanálisis en los círculos intelectuales y científi$\cos ^{12}$.

Alrededor del año treinta, llega también a Buenos Aires la traducción de la obra de Freud por López Ballesteros. En 1933, Emilio Pizarro Crespo publica en la revista Archivos argentinos de psicología normal, un trabajo en el que intenta explicar el narcisismo como una enfermedad de nuestra sociedad. En 1934, Enrique Pichón Riviere había creado una sección de psicoanálisis en la revista Nervio. En 1936, la revista Psicoterapia, dirigida por Gregorio Berman, dedica su número de septiembre a Freud: escribían allí Pizarro Crespo y Angel Garma, éste último aún desde el exterior. Por esta época aparece un libro de Fernando Gorriti, que cuenta la curación de un síndrome de posesión psicótico por medio del análisis de los sueños. Esa obra resultó del mayor interés para Celes Cárcaomo, futuro pionero del psicoanálisis en el país, quien en 1936 decide trasladarse a París para trabajar en el Instituto Psicoanalítico. En 1937, Arnaldo Rascovsky funda un grupo de estudio freudiano que se reúne en su casa, en el que participaban su esposa Matilde, Luisa Gambier, Simón Wencelblat, Flora Scolni, : Alberto Tallaferro, Teodoro Schlossberg y Guillermo Ferrari Hardoy. Bela Székeli llega a la Argentina en 1938; húngaro de origen, se había licenciado en psicología en 1932, y es quien publica la primera monografía en castellano sobre Rorschach. Emilio Mira y López, en 1940, en la Facultad de Medicina, habla de la necesidad de una comprensión psicosomática de esa disciplina ${ }^{13}$.

En el clima cultural de Buenos Aires donde se desarrolla el incipiente movimiento psicoanalítico argentino se asiste a la contienda civil española, de grandes repercusiones en nuestro medio, y a los prolegómenos de la segunda guerra mundial, que supone también la llegada a nuestras orillas de gente del viejo continente deseosa de poder trabajar en paz.

\footnotetext{
12 E. Jones corrobora, en su texto sobre la vida y obra de Freud, que éste tenía una triple invitación para cuando deseara emigrar: Londres, EE.UU. y Buenos Aires.

${ }^{13}$ Asociación Psicoanalítica Argentina: 1942-1982. 1982, Buenos Aires, p. 8-9.
} 
En 1938 llega el español Angel Garma a Buenos Aires, que sería el factor determinante, por ser quien poseía la formación más sólida, para la constitución de la Asociación Psicoanalítica Argentina.

En enero de 1940, Arnaldo Rascovsky empieza su análisis con Garma y ocho meses después lo hace Pichón Riviere. Poco después se reúnen Garma, Cárcamo, Rascovsky, Pichón Riviere, Thénon, Székeli, Perrari Hardoy y algunos más y deciden fundar un grupo analítico cuando hubiese suficientes personas analizadas.

En 1942, Celes Cárcamo escribe a Ernest Jones, presidente de la International Psychoanalitical Association (IPA), a fin de que se acepte al grupo de estudios de Buenos Aires como componente de esta asociación.

En 1943, llega a Buenos Aires María Glas de Langer, procedente de Austria. Escribió Langer acerca de su propia experiencia previa:

«en los años treinta, en Viena, la juventud intelectual era atraída apasionadamente por el psicoanálisis y el marxismo. Hoy en día, en Buenos Aires, la juventud que conozco discute y se dedica con igual interés a estos dos grandes temas» ${ }^{14}$.

La Asociación Psicoanalítica Argentina (APA) fue fundada el 15 de diciembre de 1942. Sus fundadores fueron Cárcamo, Garma, Ferrari Hardoy, Pichón Riviere y Rascovsky. La estrategia del pequeño grupo radicado en Buenos Aires fue inteligente y redituable. En efecto, en menos de tres años, 19391942, desplegaron un movimiento hacia adentro, de consolidación y formación, y un movimiento hacia fuera: el reconocimiento de nada menos que Ernest Jones y el contacto con colegas de otros países. En 1943, en el primer número de la Revista de Psicoanálisis, financiada por un paciente de Pichón Riviere, se publica una carta de Jones de felicitación por la organización de la APA. Para la época de la carta de Jones al grupo de Buenos Aires, la suerte de la Segunda Guerra Mundial estaba echada a favor de los aliados, para mayor incertidumbre del Gobierno Argentino, el cual, a diferencia del brasileño, permanecía neutral y permitía el desarrollo de actividades nazis en su propio territorio.

En su carta, Jones comentaba:

«es de esperar que la colaboración política creciente entre los países de habla castellana e inglesa se acompañe de una correspondiente colaboración estrecha en nuestro trabajo científico..."15.

\footnotetext{
14 Langer, M. (1971): "Psicoanálisis y/o Revolución Social». Cuestionamos. Ed. Búsqueda. Buenos Aires. p. 129.

${ }_{15}$ Revista de Psicoanálisis (1943), año I, número 1. Buenos Aires.
} 


\section{APUNTES PARA UNA HISTORIA DEL PSICOANALISIS EN ARGENTINA}

Algunos comentarios: Argentina era una semi-colonia económica británica. El desafío alcanzaba al terreno intelectual. ¿El pequeño grupo de Buenos Aires podía prescindir de un Gran Otro o funcionaría como un apéndice semicolonial? Dentro de los factores de poder, las fuerzas estaban ya divididas antes del golpe militar del 4 de junio de 1943, que dio por tierra con el gobierno conservador fraudulento. En efecto, echando una mirada retrospectiva a aquellos años en los cuales se originó la APA, podemos colegir que parte del ejército simpatizaba con el Eje, más específicamente el denominado "sector nacionalista» de éste, protagonista principal del golpe militar antes mencionado. La fuerza aérea era por entonces poco significativa. La Marina tenía como referente principal a la Real Marina Británica. La Sociedad Rural comerciaba libremente con el Reino Unido. Los frigoríficos y la red ferroviaria levantada por los ingleses llevaban las mercancías al puerto de Buenos Aires. Ciudades enteras, como Ensenada y Berisso, en la provincia de Buenos Aires, sustentaban su esquema laboral-económico en los frigoríficos Swift y Armour que llegaron a tener cerca de 20.000 operarios. En algún sector industrial y en ciertos clubes alemanes de "beneficencia» existían adhesiones al nacionalsocialismo alemán, si bien eran minoritarios en el conjunto de la población.

Las palabras del Presidente de la IPA no eran inocentes, no sólo se trataba de la adhesión a la teoría freudiana, sino también de la cuestión de la colaboración política entre la potencia imperial británica y la semi-colonia y su puñado de adherentes. No obstante, no debe hacerse excesivo hincapié en este intercambio epistolar, ya que el grupo argentino debió esperar el reconocimiento internacional hasta 1949.

Las primeras reuniones de la APA se llevaron a cabo en la casa de Celes Cárcamo. Más adelante, la Sociedad Científica Argentina ofreció su sede y, pocos meses después, la APA pudo alquilar su primer local en Juncal $655,1 .^{\circ} \mathrm{B}$.

Los fundadores se comprometieron a presentar un trabajo científico antes de un año, condición ésta que los habilitaría para su propia promoción. En efecto, los fundadores ingresaban en la naciente institución como adherentes «reconocidos de facto» por Jones, vale decir, por la Asociación Psicoanalítica Internacional, IPA. Al poco tiempo, trabajo mediante, eran ascendidos a miembros titulares. ¿Quiénes podían ser los miembros adherentes, desde diciembre de 1942? Según texto de la APA: «los miembros adherentes, se convino, serían las personas que tuvieran una formación psicoanalítica consistente en un psicoanálisis didáctico terminado, en varios psicoanálisis controlados y en alguna contribución científica psicoanalítica presentada a la Asociación» ${ }^{16}$. Estas cuestiones, que conciernen a adherentes, candidatos,

16 Fundación de la APA, op. cit., cap. IV, p. 19. 
titulares, tomarán progresivamente importancia con el desarrollo de la institución, hasta el advenimiento de crisis institucionales desde mediados de los sesenta hasta 1971. El centro de la cuestión, desde mí punto de vista, pasaba por una cuestión incipiente acerca de las nupcias entre el saber y el poder, tanto dentro de la institución como en las relaciones con el exterior, en especial con los británicos - Jones-y los psicoanalistas norteamericanos.

Al comienzo de 1943, la APA contaba con 11 integrantes: 4 miembros didácticos, 3 titulares y 4 candidatos, todos de primer año. A fines de 1953, los integrantes era 68. Durante la primera década, la actividad científica, puede considerarse dividida en dos ámbitos:

1. En el interior de la APA, los cursos y seminarios de formación.

2. En el exterior de la APA, tanto a nivel internacional como nacional, en que predominan temas de medicina psicosomática.

Desde los años cincuenta de este período comienza a ser notoria la influencia de la obra de Melanie Klein, algunos de cuyos textos figuran en los primeros números de la Revista de Psicoanálisis. Los contactos directos con Angel Garma, Pichón Riviere y Arminda Aberastury, y la estadía de Hanna Segal en Buenos Aires contribuyeron a ello.

Un tema central es la producción intelectual de la APA, entre los años 1942-1953, debido a que - con excepción del psicoanálisis brasileño- la APA constituyó una revolución cultural en toda América Latina, con influencias en España, hasta bien entrada la década de los setenta ${ }^{17}$. Durante 1943-44, se publicó el primer volumen de la Revista de Psicoanálisis. La publicación de algún trabajo de E. Jones o de Anna Freud, aparentemente vitales para la función de sostén del grupo fundador, no aparecerán en el primer volumen. Jones será traducido en los años 1945-46, y Anna Freud entre 1946-47. Contrasta esta actitud con la inmediata publicación de un trabajo de M. Klein, autora que se convertirá en el Gran Otro de la institución desde la década de los cincuenta hasta su escisión, en 1971.

A comienzos de 1944, la APA publicó el Asesinato desconocido, de Theodor Reik. El gesto no deja de ser hidalgo. Reik, perseguido por el nazifascismo austriaco, tuvo que trasladarse a Berlín, para poder ejercer el análisis sin la obligatoriedad del título de médico. Garma tendía una mano al viejo maestro, que habiendo sido jaqueado en Alemania, tuvo que escapar a Holanda y de allí a EE.UU. La APA, en 1944, participó en el primer congreso de la Sociedad de Neurología y Psiquiatría de Buenos Aires. Este hecho constituye su entra-

\footnotetext{
17 Es de destacar que la primera comunicación en la historia del psicoanálisis argentino, fue el trabajo de Angel Garma: "La génesis del juicio de la realidad», presentado el 5 de marzo de 1943, en una reunión llevada a cabo en la casa de Celes Cárcamo.
} 


\section{APUNTES PARA UNA HISTORIA DEL PSICOANALISIS EN ARGENTINA}

da en el escenario científico argentino. En 1945, el presidente de la APA fue invitado a Río de Janeiro y San Pablo, comenzando la búsqueda de adherentes en Sudamérica. En este momento histórico del psicoanálisis argentino, el pequeño grupo fundador de la APA llevaba a cabo una actividad intelectual que colocaba a la Argentina, a pesar de sus propios conflictos políticos internos, por encima de Francia. Sólo Inglaterra y los EE.UU. mantenían un nivel superior.

En 1946, llegó a Buenos Aires el primer miembro de la Asociación Psicoanalítica Norteamericana, Gregory Zilboorg. El mismo año se desarrolló el I Congreso Interamericano de Medicina, en el que estuvieron presentes la casi totalidad de los miembros de la APA. En los trabajos locales, las líneas de acercamiento tendían hacia la psiquiatría y hacia los temas psicosomáticos. En el plano local los sucesos invitaban a la prudencia del grupo de la APA. Recordemos aquellas palabras de Marie Langer:

"los EE.UU. caen en el macartismo. En la Argentina sube Perón. De nuevo no hay que llamar la atención ni meterse en la izquierda. Hay que mantenerse quieto, para que la joven Asociación Psicoanalítica no corra peligro» ${ }^{18}$.

Es importante destacar que en 1949 el hecho más relevante fue la participación de la Asociación en el XVI Congreso Psicoanalítico Internacional realizado en Zurich. En dicha reunión se confirmó la afiliación de la APA a la International Psycho-Analytical Association. Ese mismo año, un miembro de la APA, el Dr. Eduardo Krapf, fue designado Consultor Experto en Salud Mental de la Organización Mundial de la Salud.

En 1950, Enrique Pichón Riviere fue elegido presidente de la Sociedad de Psicología Médica, Psicoanálisis y Medicina Psicosomática. La estrategia era ganar la confianza de los médicos, para no padecer hostilidades gratuitas ${ }^{19}$. Si la estrategia era no tener a los médicos como enemigos, la táctica era entrar en la Asociación Médica Argentina, cosa que se consiguió en 1950, lejos de la égida de la Sociedad de Neurología y Psiquiatría, la cual pretendía englobar al psicoanálisis; hecho que hubiera detenido su desarrollo. La táctica, como he comentado, consistía en una profusa producción que tendía hacia lo psicosomático. Esta producción hermanaba a la joven APA con la medicina y por otra parte con la Sociedad Psicoanalítica Norteamericana, que seguía esa vertiente científica.

\footnotetext{
18 LANGer, Marie (comp.) (1971): Cuestionamos. Plataforma-Documento. Ruptura con la APA. Buenos Aires. Ed. Búsqueda, p. 133.

19 Recordemos la incómoda posición de Freud en Viena desde los orígenes del psicoanálisis y su ruptura epistemológica con el Poder Médico.
} 
Es interesante destacar el hecho de que Jacques Lacan estuvo presente en aquel XVI Congreso Internacional, donde presentó su trabajo «Le stade du miroir comme formateur de la fonction du Je telle qu'elle nous est révélée dans l'experience psychanalytique». Durante el Congreso Pichón Riviere entabló amistad con Lacan ${ }^{20}$.

En 1954 se produce un hecho de importancia: varios miembros de la APA asistieron al V Congreso de Higiene Mental y I Congreso Internacional sobre Psicoterapia de Grupo, realizado en Toronto, Canadá. En 1955, Jorge Mom, J.J. Morgan, Raúl Usandivaras, León Grinberg, Marie Langer, J.L. Muratorio, Salomón Resnik, Emilio Rodrigué, G. Royer, J. Puget y Madelaine Baranger, fundaron la Asociación Argentina de Psicología de Grupo que organizó al año siguiente el Primer Congreso Latinoamericano de Psicoterapia de Grupo ${ }^{21}$. A través de este acto inaugural de apertura hacia la terapia grupal analítica, tuvo ésta un desarrollo singular en la Argentina, en especial en la década siguiente y en parte de los setenta, hasta el advenimiento de la dictadura militar dirigida por el General Videla. Esta forma de terapia, como otras derivadas del psicoanálisis, fueron barridas de la escena institucional (hospitales, Universidad, etc.) desde 1976, especialmente, hasta la recuperación de la democracia a fines de 1983. Durante este tiempo aparece lo que he denominado Discurso único hegemónico (y en la actualidad, post-lacaniano). Ese mismo año, en diciembre, Willy y Madelaine Baranger ${ }^{22}$, se trasladaron a Montevideo, como enviados de la APA para formar al incipiente grupo psicoanalítico uruguayo.

Hacia mediados de la década de los cincuenta se producían diversos sucesos que impactaron al psicoanálisis argentino, alrededor del hecho político de la caída del gobierno de Juan Domingo Perón. Se habilitaron nuevas carreras universitarias: Arquitectura y Psicología fueron las más importantes. La carrera de Psicología, primero en la Universidad Nacional de Buenos Aires y luego en el interior - vrg. La Plata- se nutrió de profesores a menudo surgidos de la APA. La Universidad de Buenos Aires, a través de los sectores que apoyaban al rector Risieri Frondizi promovieron un acercamiento entre la APA y la Facultad de Medicina. Así, dictaron clases en la Universidad, entre 1957 y 1960, profesores del APA tales como Marie Langer, Luis Rascovsky y Angel Garma entre otros.

\footnotetext{
20 "Creo que Lacan me sintió «lacaniano", así como yo le sentí "pichoniano». No somos ni lo uno ni lo otro, pero Freud, el surrealismo y la cultura francesa fueron las claves de una amistad que permanece inalterable con el tiempo".

Entrevista a Enrique Pichón Riviere. Revista Actualidad Psicológica (1975), año I, N. ${ }^{\circ} 12$, p. $1-9$.

21 APA 1942-1982 op. cit., p. 42.

22 Willy Baranger, de origen tunecino-francés, integrará hacia fines de los sesenta el IVème Groupe, en París. El grupo se separó de Lacan cuestionándolo más como persona que como teórico.
} 


\section{APUNTES PARA UNA HISTORIA DEL PSICOANALISIS EN ARGENTINA}

Comenzados los sesenta, surgirán, dentro del APA, distintos grupos que se encolumnarán detrás de distintos maestros. Uno de ellos será el de Marie Langer, quien a fines de 1971 participará del grupo denominado «Plataforma», cuyos integrantes "determinaron una ruptura institucional» por la que dejaron la APA unos 30 miembros y unos 20 candidatos del Instituto ${ }^{23}$. Amenazada de muerte por la triple A (Alianza Anticomunista Argentina) Langer partirá a mediados de los setenta hacia México, desarrollando una intensa actividad en distintos países de América Latina.

Como ya he afirmado, desde la década de los cincuenta Melanie Klein se constituye, para la mayor parte de los analistas argentinos, en el punto de referencia obligado, teórico y práctico, durante dos décadas. Klein fue, quizás a pesar de sí misma, un Gran Otro para distintas generaciones de psicoanalistas, hasta cerca de 1970. Lacan, su sucesor en nuestro país en el lugar imaginario de Gran Otro, probablemente buscase ex-profeso ese lugar, paraíso soñado en el cual se entremezclan incesantemente (para el análisis argentino) saber, poder y... dinero. Las obras de Klein se constituyeron rápidamente en una suerte de "Biblia» para todo aquél que se preciara de buen analista. No se podía pensar el psicoanálisis sin tener en cuenta la revolución kleiniana. A pesar de ello, la publicación de sus obras completas, en 1974, fue tardía. Analistas de la talla de Pichón Riviere y su esposa, Arminda Aberastury, pionera del psicoanálisis de niños en nuestro país, controlaron su tarea con Melanie Klein. Emilio Rodrigué trabajó a su lado.

Hacia 1970, leer a Freud parecía antiguo...

Por el contrario, el escaso entusiasmo de la obra de Anna Freud no dejaba de ser extraño en contraposición a la actitud de los psicoanalistas en relación a los colegas norteamericanos, fuertemente influenciados por aquélla. Esta situación paradójica fue puesta al descubierto en julio de 1966. En efecto, algunos pensadores como Heinz Kohut - centroeuropeo, emigrado a los EE.UU.constituyeron el centro de atención del II Congreso Panamericano de recepción, en julio de 1966, en la ciudad de Buenos Aires, a pocos días del golpe militar que derrocó al presidente radical Arturo Illía. Kohut fue la estrella del Congreso. Los argentinos eran todos kleinianos, pero admiraban a los colegas del Norte, «hijos» o «nietos» de Anna Freud, dejada de lado en Buenos Aires.

\section{Los nuevos tiempos}

El comentario sobre la situación de la obra de Anna Freud me ha llevado a relatar la posición de los psicoanalistas de la APA, desde fines de la década

\footnotetext{
${ }^{23}$ LANGER, Marie (comp.) (1971): Cuestionamos. Plataforma-Documento. Ed. Búsqueda. Buenos Aires.
} 
de los cuarenta a julio de 1966. Lo precedente tiene como objeto poner de relieve el deslizamiento teórico producido durante esos años, desde la obra de Sigmund Freud, en los pioneros, hasta la adopción del kleinismo como dogma. Sobre este terreno, ideal para la búsqueda de «Hermanos mayores», en el sentido orwelliano del término, se asentarán en años venideros -y aún contemporáneos- Karl Marx y Jacques Lacan.

"Para un peronista no hay nada mejor que otro peronista», reza una de las veinte verdades de la doctrina nacional-justicialista. Esta ley del todo o nada en el terreno de las ideas políticas y de su praxis tenía y tiene correlato en el campo psicoanalítico, a pesar de su asepsia política hasta los sucesos nacionales que se fueron desarrollando a partir de mayo de 1969 con el Cordobazo.

"Para un kleiniano no hay nada mejor que otro kleiniano", podía haber sido el slogan psicoanalítico entre la década del cincuenta y la del sesenta...

«Para un marxista...etc», en los primeros setenta.

"Para un lacaniano...etc», desde mediados de los setenta hasta la actualidad.

La ley del todo o nada, que atravesó a la sociedad argentina a lo largo de su historia, también penetró en la institución psicoanalítica. La necesidad de un Big Brother, de un Peter Patrum, de un líder, ha sido una constante en la historia argentina contemporánea, historia de la que no ha podido escapar el psicoanálisis ni los psicoanalistas. Este tema, el tema del Big Brother es - desde mi punto de vista- una zona de entrecruzamientos entre aspectos de la historia argentina (Vgr. las historias de Hipólito Yrigoyen y Juan Domingo Perón, mas allá de sus trayectorias políticas y anhelos) y la historia del psicoanálisis argentino.

Parafraseando al político Moisés Lebenshon en su cuestionamiento a la reforma constitucional argentina de 1949, podría decir: "Qué de distinto ocurre en nuestro país?». Respecto a la concepción del mundo a través de la óptica psicoanalítica, entre los precursores europeos y los psicoanalistas argentinos «desde» la posición kleiniana, o bien «desde la posición lacaniana y neolacaniana entre los años 1951-52 al setenta, Lebenshon, a través de su profunda y punzante interrogación, quería demostrar que la constitución que iba a imponerse estaba en minoría en las cámaras parlamentarias y su discurso tenía el acento ético de quien se arriesgaba por una idea aun a sabiendas de que en la votación saldrá perdedor.

Más de cuarenta años después, los que nos podíamos denominar independientes en el psicoanálisis estamos en minoría. Nos queda como tarea levantar las banderas antiautoritarias en el terreno del psicoanálisis y tener, al mismo tiempo, la osadía anarquista - sin la connotación violenta - de oponernos a cualquier discurso que pretenda erigirse en único...

- Pluralismo en las ciencias.

- Osadía para crear.

- No sentirse bajo la égida de nadie - tal como acabo de describir. 


\section{APUNTES PARA UNA HISTORIA DEL PSICOANALISIS EN ARGENTINA}

Estos serían algunos puntos mínimos para la constitución de un psicoanálisis independiente y vivo, capaz de intercambios internacionales. No debería haber lugar para más colonización mental (colonización gustosa por parte de los propios colonizados), tampoco para autoritarismos desplazados del terreno del pensamiento, situación ésta que origina marginamientos y exilios en jornadas, congresos, etc.

Quien no era kleiniano en 1965 no era psicoanalista.

Quien no es lacaniano desde los ochenta no es analista.

O se adhiere al discurso oficial o no se es analista, o bien, si el independiente es «molesto" se transforma en adversario.

La ley del «todo o nada», ya descrita, y la búsqueda permanente del Big Brother constituyen una novedosa y a la vez antigua práctica autolimitante.

En la Argentina no existe consciencia histórica. Es un país orwelliano, en el sentido de la novela 1984. La falta de consciencia histórica trae riesgos que denunciaré de inmediato:

- El borramiento histórico (ya desarrollado) produce severas perturbaciones en la producción del pensamiento, ya sea este individual, familiar, grupal o comunitario. El borramiento histórico impide el desarrollo de tareas sensatas, tales como la construcción de una República democrática. La falta de "metabolización» de datos elementales por parte del "gran pueblo argentino" - no estoy hablando aquí de su oligarquía - produce un estado de ignorancia mal disimulada a través de la soberbia y las fantasías megalómanas. Los transmisores del conocimiento son expertos en el fenómeno del borramiento histórico. La falta de metabolización de datos elementales produce fenómenos de ensayo y error sin aprendizaje a nivel masivo, provocando por lo tanto historias circulares, cuando no una decadencia generalizada en todos los estamentos sociales. El psicoanálisis no puede escapar de los trastornos culturales que atraviesan a la sociedad en su conjunto.

- La necesidad de dependencia de un Gran Otro, ya sea éste una Nación (Inglaterra, durante el siglo XIX hasta la II Guerra Mundial; EE.UU., desde entonces), un ideal político (Stalin para el PC pre-perestroika, Hitler-Mussolini-Franco para el nazi-fascismo criollo), un líder psicoanalítico (Klein, Lacan) que enfrente a bandos opuestos.

En la Argentina no se debate nada:

- Se manda. Se obedece. Puede haber rebeldías violentas.

- Se copia. Se depende. Se malgasta el tiempo, las riquezas humanas y materiales. Se dilapida el poder hacer. 
- Se despilfarra la creatividad. No se lucha por causas justas. La palabra solidaridad ha sido borrada de las mentes. Se subsiste. El adversario es un enemigo.

- Está todo por transformarse, pero un raro "opio" paraliza el propio pensamiento hasta la exasperación.

- No se debate nada. No hay riqueza de pensamiento.

- No se fomenta la multiplicidad de modelos creativos. Hay carencias de modelos positivos. Hay competidores vernáculos, que desde el interior de una oficina pública hasta un consultorio, o desde grandes despachos alfombrados, con sables o sin ellos, repiten slogans que vienen de territorios alejados. Los slogans provienen de Grandes Otros que a veces ni siquiera conocen la Argentina o no les interesa ocupar el lugar de un Big Brother (pienso en Melanie Klein, o más recientemente en Piera Aulagnier). Von Hayek, Milton Friedman, en economía, dan su perfil de neoliberalismo económico para uno de los países más frecuentemente endeudados. Lacan para los llamados intelectuales... y no le pidamos más a esta Argentina que alguna vez pudo ser uno de los países importantes del orbe y ahora está confrontada, como Dorian Gray, con su propio retrato.

\begin{abstract}
«El lacanismo ha llenado espacios y ha promovido un tipo muy particular de personaje en nuestro medio: el que garantiza la teoría. Ese personaje solo existe en la Argentina. Es un señor que no es psicoanalista, ni se psicoanalizó nunca, que nunca "pudo jugarse sobre el diván", que puede ser muy inteligente, muy estudioso y que se transforma en garante de una teoría porque, como en la práctica, por definición, no hay garantías, hay que acudir a alguien que garantice saber. En Francia, además, se discuten ideas, aquí no, aquí es el dogma, aquello que yo digo transforma al otro instantáneamente, en perjuro o en adepto. No se puede discutir ideas. Esto en psicoanálisis parece que lo hubiésemos heredado de esa burguesía que manejó nuestro país ${ }^{24}$.
\end{abstract}

Hubo una desaparición casi completa del amplio abanico teórico y práctico del psicoanálisis argentino de los sesenta y parte de los setenta, sincrónica con la aparición de la parapolicial triple A (1975) y con el golpe militar de Videla en marzo de 1976. A partir de estas fechas, no sólo desaparecerían personas, sino también lugares, facultades, universidades, enseñanzas.

Cuando comparo estos dos fenómenos, a saber: desaparición de personas, instituciones, teorías y prácticas, por el activismo de las bandas parapoliciales y paramilitares (1975) y luego por el sistemático terrorismo de Estado a partir de marzo de 1976; con el crecimiento y desarrollo de decenas y hoy

\footnotetext{
${ }^{24}$ Revista Territorios (1986): «Reportaje a Diego García Reynoso», N. 3, Buenos Aires.
} 


\section{APUNTES PARA UNA HISTORIA DEL PSICOANALISIS EN ARGENTINA}

(1990) centenas de instituciones lacanianas que se escudan debajo del apellido «Freud» (Vgr. Escuela Freudiana de Buenos Aires, Círculo Freudiano, Casa Freudiana, etc.), soy consciente de que ambos son sincrónicos, pero que no son vinculantes. La práctica del terror estatal desmanteló instituciones, acalló publicaciones y envió al exilio o al cementerio a miles de argentinos. Se produjo un vacío generacional junto al arrasamiento de cosmovisiones que prometían un país diferente. La APA se tornó muy conservadora y sufrió otra escisión en 1977, a partir de un grupo denominado El Ateneo, que formaría la Asociación Psicoanalítica de Buenos Aires (APdeBA).

Los autores y protagonistas de esta historia crítica sufrieron distintos destinos:

- Eduardo Pavlovsky emigraría a España en 1978 para retornar hacia 1980.

- Hernán Kesselman también emigraría a España hasta 1986. Reside en Buenos Aires.

- Armando Bauleo se iría a Italia.

- Emilio Rodrigué exiliado en Brasil.

- Marie Langer vivió en México desde 1974. Realizó una intensa labor docente en la Nicaragua Sandinista desde 1980. Hizo presente el psicoanálisis en la reacia Cuba en 1985. Falleció en 1987.

- Los hermanos Gregorio y Valentín Barenblit emigraron alrededor de 1975.

- Diego García Reynoso, fallecido, se exilió en México.

Todos los mencionados estaban ligados al grupo Plataforma.

- Isidoro Berenstein permaneció algún tiempo en Israel, desde 1977. Reside actualmente en Buenos Aires y es uno de los analistas más creativos.

- José Bleger murió a los 49 años en Buenos Aires, en 1972.

- Arminda Aberastury murió el 13 de noviembre de 1972.

- Enrique Pichón Riviere murió en Buenos Aires en 1977.

- Hugo Bleichmar emigró a Venezuela. Actualmente reside en España.

- Luis Hornstein haría otro tanto, regresando a Argentina entre 1987-88.

- Hugo Francisco Bellagamba figura en los Anexos del Informe de la Comisión Nacional sobre la desaparición de personas con el número 00523, secuestrado el 19 de abril de 1976.

- Beatriz Perosio, presidenta de la Asociación de Psicólogos de Buenos Aires, figura en el listado precedente de personas desaparecidas con el número 04002. Secuestrada el 8 de agosto de 1978, a sesenta días de iniciado el Mundial de Fútbol. 


\section{HORACIO R. MARIN}

- Mauricio Goldenberg también partiría hacia el exilio, hasta el día de la fecha, a excepción del apoyo brindado al Gobierno Constitucional a partir del 10 de diciembre de 1983. El plan Nacional de Salud Mental, llevado a cabo - como se pudo- por Vicente Galli en la Nación y por mí mismo en la Provincia de Buenos Aires, llevaban su importancia.

- León Grinberg marchó a Madrid.

Hago un punto en la larga lista de exiliados, muertos, desaparecidos...

Existió una profunda diferencia entre el antes y el después de los hechos que acabo de describir.

La Universidad, tanto en la Capital como en el interior, estaba en manos de fascistas desde 1974. La carrera de Psicología fue cerrada en la Universidad de La Plata por «difusión de ideas disolventes ajenas al ser nacional», y muy limitada su actividad en otras universidades. Pasó a enseñarse Psicología predominantemente en Universidades católicas, que incluían Teología durante cinco años. Freud pasó a ser cuestionado, como también Piaget. Pero el centro de la persecución pasaba por el activismo político de la víctima, o por su supuesto activismo.

En medio de la devastación nazifascista proviniente de los militares argentinos y de sus socios conservadores, los psicoanalistas nos replegamos de los espacios políticos y públicos hasta 1983. En innumerables grupos de estudio, que muchas veces sustituían a la cátedra universitaria, se instauró lo que alguien denominó "cultura de las catacumbas». Y, desde diversos lugares, en todo el país pero en especial en las ciudades de Buenos Aires, La Plata, Rosario y otras, comenzó a circular la críptica obra de Lacan. Estoy hablando del período 1976-80.

Lo que no pudieron lograr ni Maud Mannoni ni Serge Laclaire, desde el comienzo de los setenta, como tampoco Oscar Masotta ${ }^{25}$, hacia fines de los sesenta, pudieron hacerlo decenas y luego centenas de «didactas» que no tenían -en su mayoría - contacto con la APA. El lacanismo, una corriente más entre todas las teorías y prácticas que estaban presentes en la Argentina en los primeros setenta, quedó en una posición privilegiada ante la desaparición de instituciones y personas.

En 1975 y también 1976 era leído como texto básico La comunicación en terapéutica psicoanalítica, y su superación: Lingüística, interacción comunicativa y proceso psicoanalítico, de David Liberman ${ }^{26}$. Un proceso de «olvido»

\footnotetext{
25 Osacar Masotta, un ensayista que no era psicoanalista, es el introductor de la obra de Lacan en Argentina.

26 LIBERMAN, David (1962): La comunicación en terapeútica psicoanalítica. Ed. Eudeba. Buenos Aires; y (1972) Lingüistica, interacción comunicativa y proceso psicoanalítico. Nueva versión. Buenos Aires.
} 


\section{APUNTES PARA UNA HISTORIA DEL PSICOANALISIS EN ARGENTINA}

produjo el borramiento histórico de Liberman, el acrecentamiento de la figura de Masotta, y la hipertrofia de la enseñanza de Lacan, que seguía siendo una suerte de jeroglífico alejado del campo de la Salud Mental. ¿Por qué jeroglífico? Porque las traducciones que siguieran a sus Ecrits tardarían años en aparecer.

Alrededor de la muerte de Lacan, la disolución de la Escuela Freudiana de París y la creación de la Causa Freudiana, casi como emblema de batalla de la Fundación del Campo Freudiano, han sucedido demasiadas cosas, en París y en Buenos Aires, sobre todo en lo que se refiere a la comercialización de la producción intelectual de Jacques Lacan. La profusión de bataholas, juicios penales, escisiones de los lacanianos durante toda la década del ochenta y lo que del noventa ha transcurrido, me hicieron sentir más cómodo por otros territorios, que eran próximos al concepto de salud mental y no renegaban de la palabra psicoterapia ${ }^{27}$.

\section{A modo de conclusión}

El psicoanálisis en la Argentina surgió durante la Segunda Guerra Mundial, con el acto inaugural de su fundación hacia fines de 1942, año de la creación de la Asociación Psicoanalítica Argentina. La obra de los pioneros puso en funcionamiento al movimiento quizás más extenso de habla hispana en la actualidad.

El psicoanálisis argentino tuvo un comienzo freudiano, con simpatía hacia lo que Sigmund Freud denominaba «Psicoanálisis Aplicado», como también ligámenes con la Medicina Psicosomática. El primer gran referente de los pioneros era el propio Freud.

En los años cincuenta comienzan los debates acerca de cuestiones técnicas, al mismo tiempo que se fue acrecentando la importancia de Melanie Klein. La tendencia kleiniana tuvo su pico máximo en los sesenta. El proceso de politización aguda que tuvo el país hacia fines de esos años permitió una variedad de técnicas y teorías: terapias de pareja, grupo, familia. Lo interdisciplinario se tornó una exigencia. No todos los fenómenos podían explicarse desde la problemática edípica. Se experimentaron distintas variantes terapéuticas: la coterapia, la psicoterapia breve, la asociación farmacológica, la comunidad terapéutica, etc. Jaques Lacan apenas figuraba en los intereses de pequeños grupos.

\footnotetext{
27 Remito al lector a la obra de Piera Aulagnier, una de las primeras disidentes de Lacan Con ella he mantenido una relación de amistad y de trabajo durante una década: 1979-1989. Su muerte, en marzo de 1990 está demasiado próxima y a pesar de que hagamos homenajes a su pérdida es irreemplazable, tanto en lo humano como en lo teórico.
} 
En los setenta, con el aumento de la represión política y sus consecuencias sobre toda la comunidad -aquí psicoanalítica-, existió un fenómeno prototípico de la historia argentina: el fenómeno de borramiento histórico. El psicoanálisis ya era una práctica masiva, no sólo desarrollada por médicos, sino por psicólogos, a los que hacia fines de los ochenta habrían de sumárseles licenciados en sociología, en lingüística, literatos y otros.

La doctrina lacaniana se convirtió en un discurso único y hegemónico, reemplazando en un ciento por ciento a la figura de Melanie Klein de los sesenta. La necesidad de buscar un garante de la verdad «tesoro de significantes», un Gran Otro sin castraciones, un Hermano Mayor que aplaque las incertidumbres en la labor de pensar, es una necesidad hondamente arraigada en la comunidad argentina, nativa e inmigrante.

El «fenómeno Lacan» como reemplazo del «fenómeno Klein», se da en el seno de una comunidad psicoanalítica pasiva, que "quema» a sus propios creadores intelectuales en aras de lo ajeno y superior en búsqueda de una cosmovisión individualista, que busca certezas inmutables. El fenómeno del subdesarrollo y la dependencia no son sólo reflejos de situaciones económicas o resultado de pérfidas maniobras oligárquicas. El argentino medio necesita vivir bien, con seguridad. El psicoanalista medio necesita otro tanto. Quien tiene necesidades de satisfacer sus demandas de manera más o menos inmediata no hace lugar al deseo de saber - epistemofilia-, que según Freud tanto honraba, como asimismo el trabajo de pensar. La palabra alemana Arbeit figura incontables veces en las obras completas de Freud. En el psicoanalista argentino falta Arbeit y deseos de independencia, como en el argentino medio. Abunda la necesidad de sobresalir rápido y sin mayores esfuerzos.

Como no polemiza, es terreno fértil para dogmas, locales o exteriores. El dogma cierra el trabajo de pensar. Facilita el dogma, la consigna fácil, erigiéndose Grandes Otros.

Hay una clara identidad de situaciones entre sucesos de la historia nacional y la búsqueda de caudillos, con la historia del psicoanálisis argentino y su afán por los pensamientos que devienen totalitarios. Este movimiento se realiza simultánea o sucesivamente con el borramiento de toda historia anterior. El borramiento histórico se convierte en una suerte de mecanismo de defensa colectivo que "cierra los ojos" y deforma la realidad. Cada ciudadano, psicoanalista o no, sabe más de lo que dice. Simplemente se cuida, callándose la boca y guardando secretos por las dudas. Las nuevas generaciones, como quizás ocurra en otros lugares del mundo, no cultivan el gusto por la historia y se ven expuestas a repetir errores.

Dependencia, sumisión a un Gran Otro omnipotente, borramiento y falsificación de la historia caracterizan a una comunidad muy perturbada. El psicoanálisis argentino, que alguna vez se denominó Escuela Argentina, corre el peligro de extinción por la pasión de parecerse a... 